\title{
Gene Repertoire of Amoeba-Associated Giant Viruses
}

\author{
Philippe Colson ${ }^{\mathrm{a}, \mathrm{b}}$ Didier Raoult ${ }^{\mathrm{a}, \mathrm{b}}$ \\ a URMITE UMR CNRS 6236 IRD 198, Facultés de Médecine et de Pharmacie, Université de la Méditerranée, \\ et ${ }^{\text {b }}$ ôle des Maladies Infectieuses et Tropicales Clinique et Biologique, Fédération de \\ Bactériologie-Hygiène-Virologie, Centre Hospitalo-Universitaire Timone, Marseille, France
}

\author{
Key Words \\ Acanthamoeba polyphaga mimivirus · Core genes · Gene \\ duplication - Giant virus $\cdot$ Lateral gene transfer . \\ Marseillevirus $\cdot$ Mimiviridae $\cdot$ Nucleo-cytoplasmic large \\ DNA viruses $\cdot$ Virophage
}

\begin{abstract}
Acanthamoeba polyphaga mimivirus, Marseillevirus, and Sputnik, a virophage, are intra-amoebal viruses that have been isolated from water collected in cooling towers. They have provided fascinating data and have raised exciting questions about viruses definition and evolution. Mimivirus and Marseillevirus have been classified in the nucleo-cytoplasmic large DNA viruses (NCLDVs) class. Their genomes are the largest and fifth largest viral genomes sequenced so far. The gene repertoire of these amoeba-associated viruses can be divided into four groups: the core genome, genes acquired by lateral gene transfer, duplicated genes, and ORFans. Open reading frames (ORFs) that have homologs in the NCLDVs core gene set represent 2.9 and $6.1 \%$ of the Mimivirus and Marseillevirus gene contents, respectively. A substantial proportion of the Mimivirus, Marseillevirus and Sputnik ORFs exhibit sequence similarities to homologs found in bacteria, archaea, eukaryotes or viruses. The large amount of chimeric genes in these viral genomes might have resulted from acquisitions by lateral gene transfers, im-
\end{abstract}

plicating sympatric bacteria and viruses with an intra-amoebal lifestyle. In addition, lineage-specific gene expansion may have played a major role in the genome shaping. Altogether, the data so far accumulated on amoeba-associated giant viruses are a powerful incentive to isolate and study additional strains to gain better understanding of their pangenome.

Copyright $\odot 2010$ S. Karger AG, Basel

\section{Introduction}

Viruses are ubiquitous components of the biosphere, outnumbering cells by at least 10 -fold, and they have extremely variable genomes in terms of composition, organization, and size $[1,2]$. Nevertheless, they have not been incorporated into the universal tree of life $[3,4]$, are not considered living beings according to the International Committee on Taxonomy of Viruses [5], and are still defined on the basis of negative criteria and as specialized and simple organisms $[6,7]$.

The recent discoveries of giant viruses associated with amoebae have strongly challenged this paradigm [8-11]. Acanthamoeba polyphaga mimivirus (APMV) is the first member of Mimiviridae, a new family of nucleo-cytoplasmic large DNA viruses (NCLDVs) that form a monophyletic class on the basis of a small subset of about 41 con-

\section{KARGER}

Fax +4161306 1234

E-Mail karger@karger.ch

www.karger.com
(C) 2010 S. Karger AG, Basel

$0300-5526 / 10 / 0535-0330 \$ 26.00 / 0$

Accessible online at:

www.karger.com/int
Didier Raoult, MD, PhD

Unité des Rickettsies, URMITE UMR CNRS 6236 IRD 198

Faculté de Médecine, Université de la Méditerranée, 27 Boulevard Jean Moulin

FR-13385 Marseille Cedex 05 (France)

Tel. +33 491324 375, Fax +33 491387 772, E-Mail didier.raoult@gmail.com 
served genes [12]. It is currently the largest known virus $(0.8 \mu \mathrm{m})$, being larger than some bacteria, and has a $1.18-\mathrm{Mb}$ genome that encodes more than 100 proteins [9]. Moreover, some of these proteins are components of the translation machinery never before found in viruses. A second virus, Sputnik, is a parasite of a new strain of APMV [8]. It is $50 \mathrm{~nm}$ in size and cannot be classified in a known viral family. Due to its functional analogy with bacteriophages, it has been named a virophage. Another giant virus that has been classified into a new viral family of NCLDVs, the Marseillevirus, was isolated using amoebae from a cooling tower [11]. It is the fifth largest viral genome sequenced so far. Here we review data about the content, origin, and significance of the gene repertoire of amoeba-associated giant viruses.

\section{Mimivirus, Sputnik and Marseillevirus Genomes}

The Mimivirus genome (GenBank accession number NC_006450.1) is a double-stranded, linear DNA molecule of $1,181,404 \mathrm{bp}$, as confirmed by restriction digests and pulse-field gel electrophoresis [9]. It is the largest among viral genomes and is, in fact, larger than that of several bacterial and archaeal parasites [13]. On the basis of the presence of two inverted repeats of approximately 900 nucleotides located near its tips, it has been hypothesized that Mimivirus may adopt a circular, Q-shaped conformation, with a long tail and a short tail formed by pairing between these repeats $[9,14]$. In contrast, the APMV genome lacks the large, terminal, inverted repeats found in the genome of phycodnaviruses, other NCLDVs $[15,16]$. The nucleotide composition of the APMV genome is $72 \% \mathrm{~A}+\mathrm{T}$, with a significant strand asymmetry corresponding to a slope reversal of the cumulative $\mathrm{A}+\mathrm{C}$ and gene excesses around position 400,000 [9]. This suggests, based on observations in bacteria [17], that the origin of replication may be located near this latter position. The number of genes transcribed from either strand is similar despite their asymmetry, with 450 and 461 'R' [(+) strand] and ' $L$ ' [(-) strand] open reading frames (ORFs), respectively, and genes tend to be transcribed away from the putative origin of replication [9]. Raoult et al. have identified in the Mimivirus genome 1,262 putative ORFs of lengths $\geq 100$ amino acid residues, of which 911 are predicted to be protein-coding genes [9]. The DNA molecule has a theoretical coding density of $90.5 \%$ and a mean intergenic space of $157 \mathrm{nt}$, thus exhibiting a genome compaction similar to that of other DNA viruses $[9,14]$. A total of 298 ORFs (24\% of the predicted genes) could be associated with functional attributes. This is a low proportion when compared to that found in the genomes of small bacteria and archaea (70\%) [18], raising questions about the origin and significance of these genes with unknown functions. Among the predicted APMV ORFs, 194 were found to match significantly with 108 clusters of orthologous groups (COGs) [9], which correspond to groups of at least three proteins from distant genomes that are more similar to each other than they are to any other proteins from the same genome [19]. Notably, this is more than twice the number of COGs represented in the genome of Paramecium bursaria Chlorella virus NY2A (PBCV-1) [20]. The 108 COGs have been linked with 17 functional categories. Interestingly, codon usage in APMV is practically the opposite of that exhibited by its amoebal host, Acanthamoeba castellanii. Thus, regarding its overall amino acid composition, the predicted APMV proteome shows a strong positive bias for residues encoded by codons rich in $\mathrm{A}+\mathrm{T}$. For instance, isoleucine, asparagine, and tyrosine are twice as frequent in Mimivirus as in amoebae or human proteins, with mimiviral frequencies of 9.9, 8.9 and 5.4\%, respectively [9].

In 2008, La Scola et al. reported the isolation of a new strain of APMV, called Mamavirus, by inoculating amoebae with water from a cooling tower located in France [8]. The Mamavirus genome is about 1,200 kbp long, and $99 \%$ of the predicted Mamavirus genes are orthologous to Mimivirus ORFs, with amino acid identity levels ranging from 75 to $100 \%$. More remarkable than the discovery of Mamavirus in amoebal cells was the concurrent observation of unknown, icosahedral, small viral particles (50 $\mathrm{nm}$ in size) in the amoebal cytoplasm of infected cells and inside the Mamaviruses factories [8]. Unexpectedly, this new virus, called Sputnik, only multiplies within A. castellanii if these cells are co-infected with Mimivirus or Mamavirus. Sputnik and Mamavirus co-infections revealed that the two viruses are produced in the same viral factory but with different locations and kinetics, Sputnik having the shorter multiplication cycle. Furthermore, coinfection of Sputnik and Mamavirus was associated with a three-fold decrease in amoeba lysis at day one post-infection and with a $70 \%$ decrease in the yield of infective Mamavirus. Concomitantly, a significant increase in the production of abnormal Mamavirus virions was noted. For instance, a six-fold increase in the thickness of the capsid was observed in some virions. Altogether, these findings indicate that Sputnik is a Mamavirus parasite that substantially affects the reproduction of its host virus. In light of its functional analogy to bacteriophages, it was classified as a virophage. The Sputnik genome is a 
circular, double-stranded DNA with a size of 18,343 bp (GenBank accession number NC_011132.1) [8]. Its organization is typical of viral genomes, showing little overlap but tight arrangement of the ORFs, and it displays a high $\mathrm{A}+\mathrm{T}$ content, similar to that observed in the APMV genome (73\%). Twenty-one predicted protein-coding genes were identified, which range in size from 88 to 779 amino acids. The classification of Sputnik as a virophage is supported by the identification of hairpin structures recently found to coincide with polyadenylation signals specifically used by the Mimivirus-encoded transcription machinery and detected at the 3 '-end of nearly all its mature transcripts [21]. A total of 16 Mimivirus-like putative hairpin structures have been identified in the Sputnik genome, located in intergenic regions in all but two cases (88\%) despite the fact that these regions only represent $20 \%$ of its genome [14]. This finding, together with the rarity of the AAAATTGA motif that is thought to be a gene promoter region specific to and highly conserved within the APMV genome [22], suggests the late expression of Sputnik genes in APMV factories [14].

Recently, a new giant virus termed Marseillevirus has been isolated on amoeba from a cooling tower [11]. Its genome is a circular, double-stranded DNA molecule of $368,453 \mathrm{bp}$. Thus, it is the fifth largest viral genome sequenced so far behind those of Mimivirus, Mamavirus, Emiliania huxleyi virus 86 (EhV86), and Paramecium bursaria Chlorella virus NY2A [20, 23]; of note, a size of about 500,000 bp was reported, on the basis of its molecular weight and sequencing, for the unpublished genome of Bacillus megaterium bacteriophage $G$ [24, 25]. The Marseillevirus genome has a $\mathrm{G}+\mathrm{C}$ content of $44.7 \%$. A total of 457 ORFs were predicted to encode proteins that range from 50 to 1,537 amino acids, the coding sequence representing $89.3 \%$ of the genome. The ORFs are equally distributed on both strands, 233 and 224 being on the negative and positive strand, respectively. For 188 ORFs, significant matches with sequence databases were obtained and/or conserved domains were identified. Phylogenetic analysis has shown that Marseillevirus represents a new viral family of NCLDVs.

\section{Gene Content of Amoeba-Associated Viruses}

The gene repertoire of amoeba-associated viruses can be divided into four groups: the core genome, genes acquired by lateral gene transfer (LGT), paralogs originating from duplication events, and ORFans (fig. 1).

\section{The Core Genome}

No common gene is shared by all viruses [26], in contrast to the situation in bacteria. Nevertheless, monophyly of five large classes of viruses and selfish genetic elements have been described based upon comparative genomics $[12,26$, 27]. Mimivirus, Mamavirus, and Marseillevirus are classified in one of them, the so-called NCLDV class. Iyer et al. first performed a comparative genomic study of NCLDVs of eukaryotes that revealed the monophyletic origin of four viral families: Poxviridae, Asfarviridae, Iridoviridae and Phycodnaviridae [27]. Thereafter, they updated their analysis by including the Mimivirus genome as well as several additional genomes of iridoviruses, phycodnaviruses and poxviruses [12]. From the most to the least evolutionarily conserved, NCLDVs core genes have been classified as class I when found in all clades, class II when they are missing in some species despite being present in all clades, class III when absent from one clade, and class IV when absent from more than one clade. In the original analysis, the NCLDVs core gene sets was found to include 9 genes shared by all families of NCLDVs and 22 additional genes shared by at least three of the four families [27]. Thereafter, it was hypothesized, based on a parsimonious reconstruction of its gene complement, that the ancestral NCLDV was a complex virus with at least 41 genes [12]. These putative genes encode proteins implicated in the replication machinery: up to four RNA polymerase subunits, at least three transcription factors, capping and polyadenylation enzymes, the DNA packaging apparatus, and structural components of an icosahedral capsid and the viral membrane. Additionally, a set of 11 conserved genes was identified for all NCLDVs, which includes genes encoding the jelly-roll capsid protein and the superfamily 3 helicase, two proteins that are among the most widely dispersed among viruses [12, 25-27]. In a very recent paper, Yutin et al. identified 47 core genes on the basis of the construction of COGs for a still extended number of NCLDV genomes, which included the Marseillevirus genome [28]; only five NCLDV COGs included proteins from all 45 analyzed viruses.

Homologs for 9/9 (100\%) class I genes, 6/8 (75\%) class II genes, 11/14 (79\%) class III genes, and 16/30 (53\%) class IV genes could be identified within the APMV genome [9]. In comparison with other NCLDVs, the COG pattern of APMV shows significant over-representation in the functional categories of translation, post-translational modification, and amino acid transport and metabolism. The APMV genome lacks two class II genes that are relevant to the biosynthesis of 3 '-deoxythymidine 5 '-tri- 


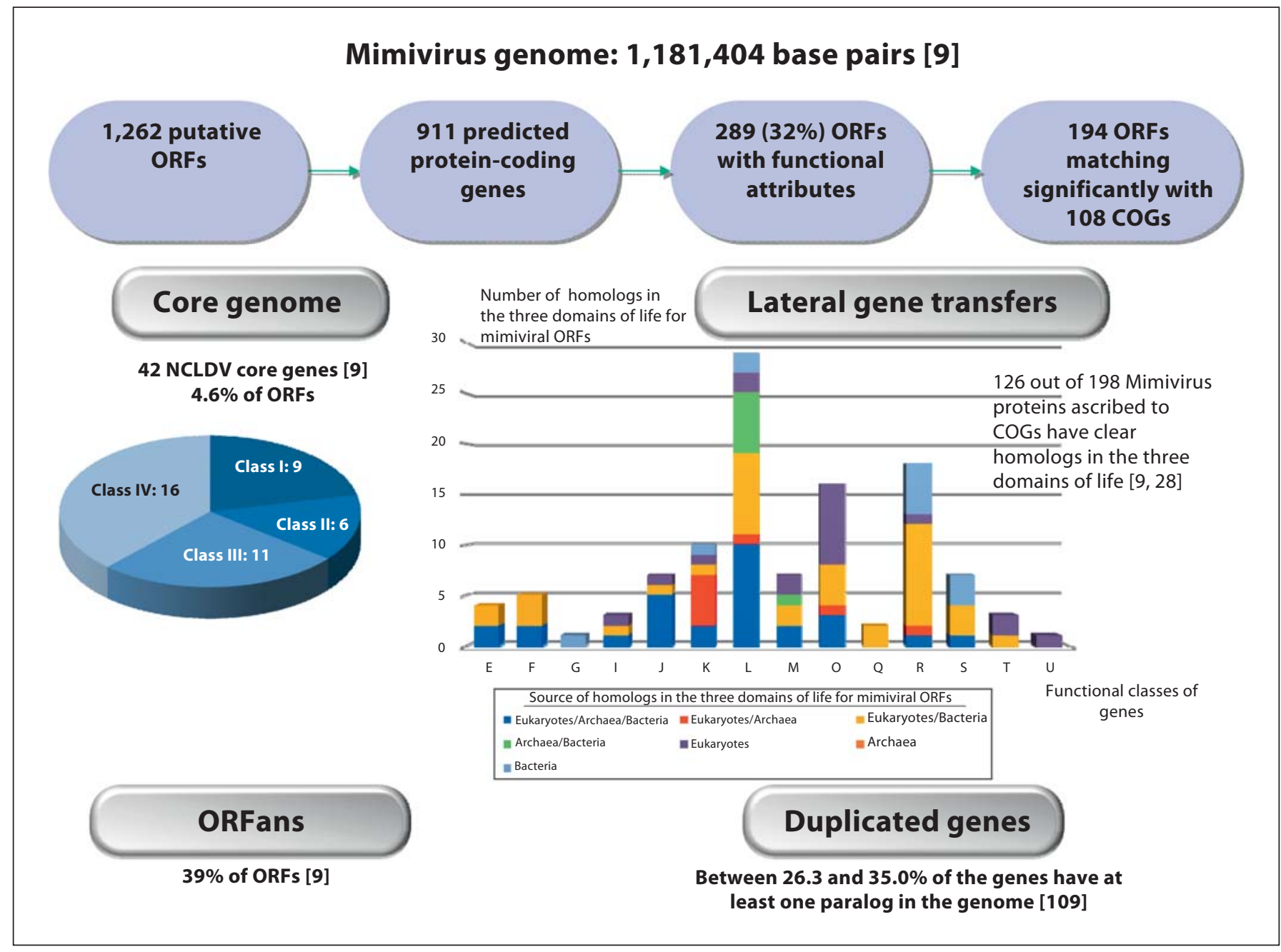

Fig. 1. Summarized representation of the gene content of the Mimivirus genome. Abbreviations for the COG functional classes: $\mathrm{E}=$ amino acid transport and metabolism; $\mathrm{F}=$ nucleotide transport and metabolism; $\mathrm{G}=$ carbohydrate transport and metabolism; I = lipid transport and metabolism; J = translation; $\mathrm{K}=$ transcription; $\mathrm{L}=$ replication, recombination and repair; $\mathrm{M}=$ cell wall/membrane biogenesis; $\mathrm{O}=$ post-translational modification, protein turnover, chaperones; $\mathrm{Q}=$ secondary metabolite biosynthesis, transport and catabolism; $\mathrm{R}=$ general function prediction only; $\mathrm{S}$ = function unknown; $\mathrm{T}$ = signal transduction mechanisms; $\mathrm{U}=$ intracellular trafficking and secretion [9]. phosphate and a class III core gene encoding an ATPdependent DNA ligase [9]. Nevertheless, it contains homologs for the class IV core genes thymidylate synthase and thymidine kinase. Additionally, the missing ATPdependent DNA ligase might have been replaced by a nicotinamide adenine dinucleotide-dependent ATP ligase also found in iridoviruses [27]. Among the additional nucleotide synthesis enzymes encoded by the APMV genome is the first nucleoside diphosphate kinase identified in a double-stranded DNA virus [9]. Moreover, it has been pointed out that with the exception of RNA polymerase subunit 10, the APMV genome harbors the same transcription-related core genes as the Poxviridae and Asfarviridae genomes. This supports the hypothesis that the transcription of at least some APMV genes is cytoplasmic [9]. Among viral transcripts detected within Mimivirus particles, three correspond to NCLDV core genes of class I or II, encoding a putative capsid protein, a DNA polymerase, and a TFII-like transcription factor [9].

In the Marseillevirus genome, 28 of the 457 ORFs are bona fide NCLDV core genes, out of the 41 previously defined classes I-III genes [11]. Six ORFs are universal NCLDV proteins, and 17 are shared with Mimivirus/ Mamavirus but are absent in other NCLDVs. Based on 
phylogenetic analysis, 51 Marseillevirus ORFs might be of NCLDV origin. As in other viruses within the NCLDV class, including APMV, the proportion of Marseillevirus ORFs that belong to the NCLDV core gene set is very small (6.1\%). Moreover, as observed for the Mimivirus genome, the core genome of Marseillevirus encodes proteins expressed and packaged in the virion particle.

\section{Genes Acquired through LGT}

Viruses are known to evolve and recombine at much higher rates than cells $[29,30]$. Moreover, they have been labeled 'gene robbers' since most viral genes involved in energy and carbon metabolism, transcription, translation and replication with cellular homologs were likely acquired by viruses through LGT, and the flux of genes from cells to viruses is thought to outweigh the flux of genes in the opposite direction [31]. Regarding APMV, it has been questioned whether it is the king among these gene robbers' [32]. The 42 ORFS identified in the APMV genome that have homologs in the NCLDV core gene set only represent $4.6 \%$ of its gene content [9]. This means that the majority of the APMV genome is lineage-specific. Recent findings indicate that lateral gene transfer and gene duplication have strongly influenced the composition of the majority of the APMV genome. However, the question of its origin remains poorly resolved, as most Mimivirus ORFs are classified as ORFans.

LGT, or horizontal gene transfer, refers to the exchange of genes between unrelated organisms [33]. It is considered a major factor of evolution in microbiology [34, 35]. For instance, LGT has had a major impact on the evolution of the genomes of bacteriophages. Their patchwork structure, made up of DNA of various and sometimes remote origins, indicates that they likely represent a substantial portion of the mobile DNA, and lysogeny is regarded as a mechanism of bacterial genomic evolution [36]. Due to the high frequency of LGT between bacteriophage families, phylogenetic boundaries may be blurred in some cases, which hampers their classification on the basis of gene content [37]. Aside from LGT between bacteriophages, LGT between bacteriophages and their host is considered a major driver of bacterial evolution, and gene subversion has provided selective advantages to phages or hosts as well [37-40]. LGT has also been studied in herpesviruses. These large enveloped DNA viruses have some of the largest viral genomes, approximately $200 \mathrm{~kb}$ comprising over 100 genes, and they are widely distributed in humans and other vertebrates [41]. Among the proteins encoded by herpesviruses, many are thought to have been acquired from their host to mimic or block normal cellular functions [42-44]. Recently, it was found that most herpesvirus proteins were clustered with those of mammals, which might reflect the co-evolution of herpesviruses with their mammalian hosts over long periods of time [45].

Almost all lineages of NCLDVs contain genes with bacterial and eukaryotic homologs [12], and the study of NCLDV non-core genes has suggested massive LGT, implicating various sources [46] (fig. 2). Noteworthy, it has been shown that genes that have homologs in host genomes tend to be clustered at the tips of the viral genome, which were identified as highly recombinogenic regions in poxviruses [47]. The main evolutionary features of NCLDV genomes have recently been summarized [48]. Only APMV and Chlorella phycodnaviruses acquired more than $2 \%$ of their genes from bacteria, the highest proportion being in APMV (9.6\%), which is twice as high as that of some phycodnaviruses. Conversely, the APMV genome has, along with Aedes taeniorhynchus iridescent virus, which belongs to the Iridoviridae family, the lowest proportion of genes acquired from their hosts. This proportion $(0.8 \%)$ is approximately one-third to one-tenth of that observed in Poxviridae. Duplicated genes constitute greater than $25 \%$ of the gene content of only four virus genomes, including the Poxviridae (Fowlpoxvirus and Ansacta moorei Entomopoxvirus) and Iridoviridae (A. taeniorhynchus iridescent virus) families, while APMV harbors the highest proportion (43\%). These data emphasize the outstanding and extreme features of the APMV genome among the NCLDVs, regarding lateral gene transfer and lineage-specific gene expansion.

Using methods that do not require inference of phylogenetic trees, it was found that 30 Mimivirus ORFs (8.3\%) out of 363 that exhibit recognizable homologs in other organisms likely originated from recent LGT [49], whereas about 40 ORFs (4.4\%) among the entire set of APMV ORFs were found to have eukaryotic or bacterial sequences as best BLAST matches [13, 50, 51]. Instead of BLAST analysis, Moreira and Brochier-Armanet specifically studied a set of 198 APMV proteins previously ascribed to COG families [9, 31, 52] (fig. 2). Among 126 ORFs for which clear homologs were retrieved, the most abundant group was ORFs present only in eukaryotes and bacteria ( $\mathrm{n}=47$ ORFs, $37 \%$ ), followed by ORFs present in all three domains $(\mathrm{n}=29,23 \%)$, and lastly ORFs present in eukaryotes only $(\mathrm{n}=21,17 \%)$. Homologous ORFs found only in bacteria $(n=12)$, in bacteria and archaea $(n=9)$, and in archaea and eukaryotes $(n=8)$ each made up less than $10 \%$ of those 126 AMPV ORFs. In addition, phylogenetic analysis inferred a eukaryotic origin 


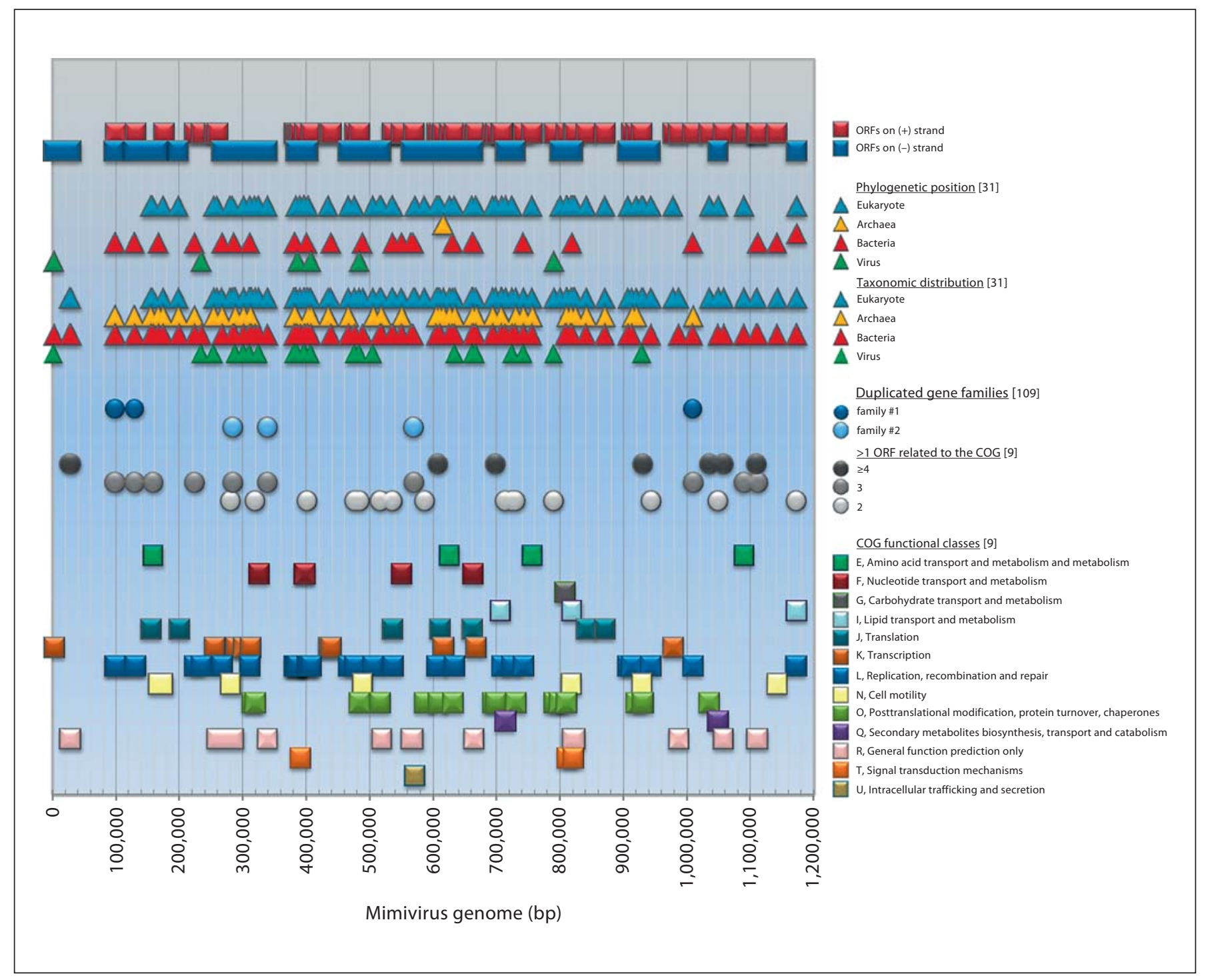

Fig. 2. Function, origin of homologs, and duplications for mimiviral ORFs ascribed to COG families. Data plotted in the figure are from the papers by Raoult et al. [9], Moreira and BrochierArmanet [31], and Suhre [109]. They take into account Mimivirus proteins ascribed to COG families and with known functions. Only proteins with homologs in cellular organisms have been analyzed. Phylogenetic positions are those that have been considered the most likely according to Moreira and Brochier-Armanet [31]. for 60 of the 126 Mimiviral ORFs that have reliable homologs in cellular species, approximately $10 \%$ of which appeared to be acquired from amoebae. A close phylogenetic relationship could be also found for a few ORFs between Mimivirus and eukaryotes unrelated to the Amoebozoa that belong to the Heterolobosea, which comprises Naegleria spp. and to the Kinetoplastida, which contains Trypanosoma and Leishmania spp. Regarding this latter phylogenetic link, it should be noted that Kinetoplastid parasites could be detected in several amoebal species
[53]. Another study unambiguously identified 96 genes within the APMV genome (compared to 48-57 within that of Chlorella phycodnaviruses) as having bacterial origin [51]. The bacterial-like genes show a strong bias in Mimivirus (and at least one phycodnavirus, NY2A) toward DNA replication and repair (20\% of proteins) and cell envelope (12.5\% of proteins) in COGs functional gene categories [51]. Other NCLDV lineages that infect metazoa or algae that do not prey on bacteria contain much fewer bacterial-like genes within their genomes, and 
Fig. 3. Intra-amoebal lifestyle as a source of complex chimeric gene contents. Colored boxes containing a $\mathrm{G}$ indicate genes from various origins (bacteria, viruses, eukaryotes).

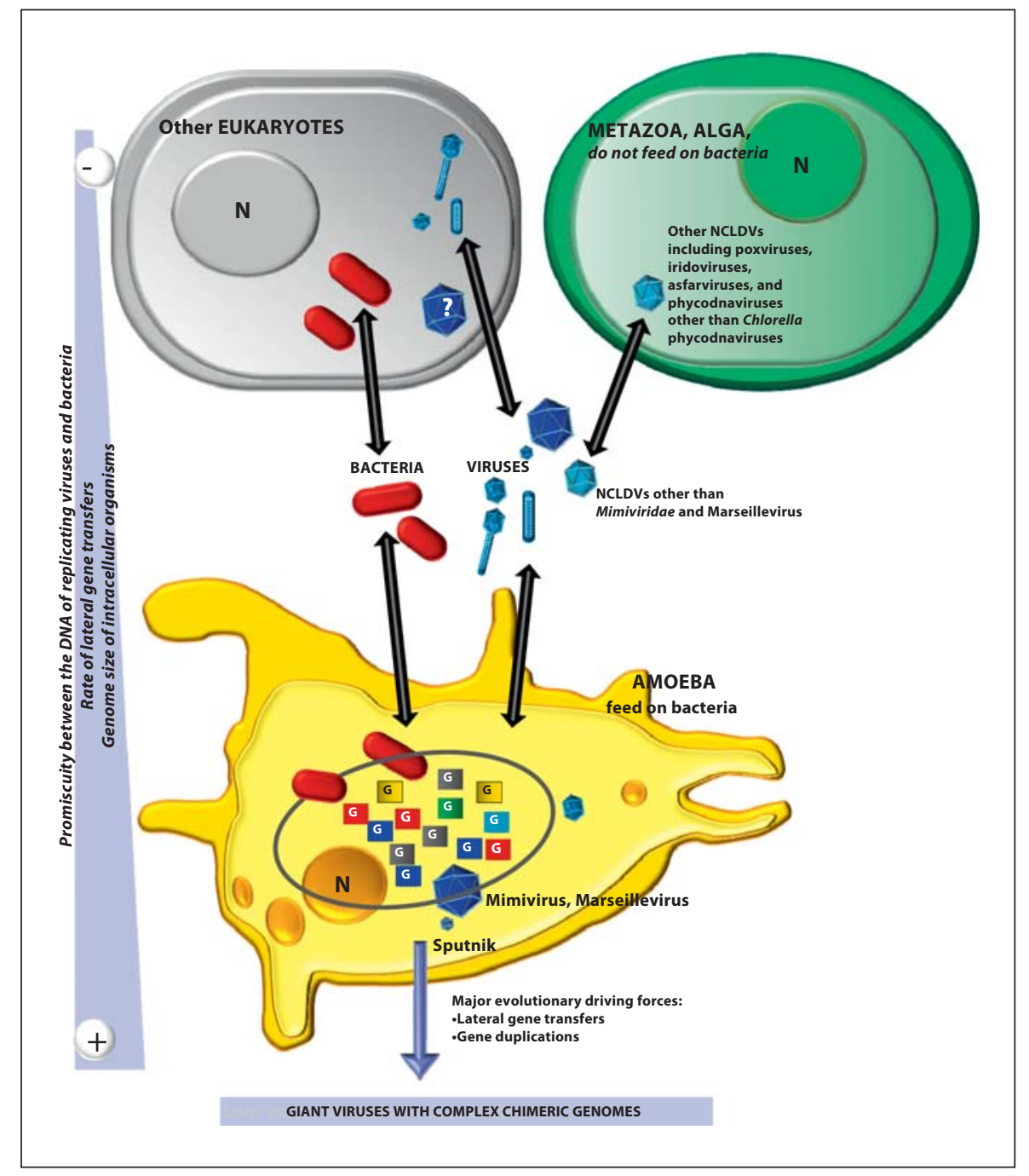

these genes are rather scattered throughout the genome [51]. These findings suggest that eukaryotic hosts using bacteria as food may provide a hotspot for the promiscuous exchange of DNA between replicating viruses and bacteria, thus providing a biological niche with access to bacterial genes (fig. 3).

Interestingly, Filee et al. examined the distribution and location of bacterial-like genes in giant viruses, including APMV [51]. They identified three consecutive co-inherited reading frames, encoding a sugar transaminase, a glycosyltransferase, and a protein of unknown function in the APMV genome, that are syntenic with three ORFs in the genome of Clostridium acetobutylicum, suggesting the inheritance of these bacterial-like genes as a short contiguous block. Additional findings also suggested co-inheritance of bacterial-like genes by NY2A, a member of the Phycodnaviridae family. Another important finding of their work was that bacterial-like genes tend to be clustered toward the extremities of the NCLDVs' genomes, possibly in islands. In the APMV genome, these regions were found positioned within the first and last $250 \mathrm{~kb}$ of the genome. In contrast, NCLDV core genes and eukaryotic-like genes are positioned toward the middle of the genome. It is noteworthy that numerous mobile genetic elements have been detected in the APMV genome, as these were previously thought to be specific to prokaryotes [51]. They include insertion sequences, considered major agents of LGT in prokaryotes [54], two homing endonucleases, and an intein. The insertion sequences contain two ORFs, one encoding a transposase and the other a protein of unknown function [55]. These two elements had previously only been identi- 
fied in the genome of one other eukaryotic virus, a brown alga virus (Ectocarpus silicosus virus 1). Those detected in the APMV genome appear to be full-length copies and belong to the IS607 family. These insertion sequences colocalize with bacterial-like genes, being embedded in some cases within stretches of contiguous bacterial-like genes (or with ORFans), further supporting their putative co-inheritance with bacterial DNA. Homing endonucleases are characterized by an $\mathrm{HNH}$ motif and are usually encoded by genes within mobile, self-splicing introns that promote the movement of the DNA sequences that encode them [56]. The two Mimivirus HNH endonuclease genes flank a unique gene typically detected in prophages of bacteria, supporting the hypothesis of acquisition by LGT from a bacteriophage [51]. Finally, an intein has been detected in the APMV genome [57]. Inteins, first discovered in 1990 [58, 59], are segments of proteins that catalyze self-splicing at the protein level. They have been found in bacteria, archaea and unicellular eukaryotes, and it has been proposed that they may be transferred between species by viruses. They have been detected in many prophages and bacteriophages, but APMV is one of the few eukaryote-infecting viruses, with iridoviruses, and Chlorella and Ostreococcus phycodnaviruses, that has been found to harbor an intein $[51,60,61]$. The Mimivirus intein is most likely to be functional and is closest to extremophilic archaeal-type inteins. Its association with a mesophilic eukaryotic-like DNA polymerase PolB gene in the APMV genome is consistent with the hypothesis that DNA viruses may have represented a central reservoir of inteins during the evolutionary course [57].

Of the 21 ORFs identified in the Sputnik genome, the 8 non-ORFan proteins have either viral, plasmidic, bacterial or eukaryotic homologs and/or homologs within the environmental Global Ocean Survey (GOS) sequence database $[8,62]$. Three ORFs are most closely related to Mimivirus and Mamavirus ORFs [8]. Among the most noteworthy features, ORF 13 contains two domains involved in viral DNA replication. Its $\mathrm{N}$-terminal domain has homologs with high similarity among proteins from the GOS database and may correspond to an archaeoeukaryotic primase, whereas its C-terminal domain is a highly conserved superfamily 3 helicase of NCLDVs. ORF 3 has limited similarity with a packaging ATPase conserved in all NCLDVs and in many bacteriophages $[62,63]$. ORF 17 encodes a protein with homologs in the GOS dataset and that belongs to the family of bacterial insertion sequence transposase DNA-binding subunits, and ORF 10 is closely related to integrases of the tyrosine recombinase family from archaeal viruses and provirus- es. The presence of these two latter ORFs within the Sputnik genome suggests that a provirophage stage may exist. This would reinforce the hypothesis of a putative role for Sputnik as a vehicle of genes. Indeed, globally, Sputnik's genomic content appears to be of chimeric origin and to be evolutionarily related to at least three distinct sources: Mimivirus/Mamavirus, an archaeal virus or a plasmid, and a putative novel family of viruses [8]. The relationship between Sputnik and Mimi-/Mamavirus genomes may be explained by gene exchange within viral factories during co-infection, as a result of recombination events. Efficient plasmid replication has been reported in poxvirus and in Asfarviridae [64, 65], supporting the hypothesis that Mimi- or Mamavirus factories may be able to replicate foreign DNA, namely that of Sputnik.

Regarding Marseillevirus, 59, 57, 70 and 2 of its predicted proteins exhibit the highest sequence similarity to viral, bacterial, eukaryotic, and archaeal homologs, respectively [11]. In 8 cases, the homologue that shows the closest similarity to the Marseillevirus ORF is from Acanthamoeba. On the basis of phylogenetic analyses, the Marseillevirus genome contains 49 genes of probable bacterial or bacteriophage origin and 85 of apparent eukaryotic origin. For 22 of these latter ORFs, a phylogenetic relationship was found with Mimivirus and Acanthamoeba ORFs. Three genes appear to originate in other Amoebozoa. Among the 188 predicted Marseillevirus ORFs, 20 encode bacterial-like membrane-occupation and recognition-nexus repeat domains, typically implicated in interactions between membranes or between membranes and cytoskeleton [66], and 10 encode bacteriophage $\mathrm{HNH}$ endonucleases and restriction-like endonucleases, which typically reside in mobile selfish genetic elements and might have been acquired by LGT. Additionally, 3 ORFs are homologous to genes encoding histone-like proteins previously found only in the genomes of two polydnaviruses (Heliothis zea virus 1, a insect nudivirus, and Cotesia plutellae bracovirus) [67, 68]. These histone proteins have been detected in the viral particle and may therefore play a role in viral DNA packaging [11]. Interestingly, the inferred origin for the predicted Marseillevirus genes tends to be non-randomly connected with their function. Thus, mixed eukaryotic and bacterial origins were inferred for genes encoding metabolic enzymes and proteins implicated in protein and lipid modification or degradation, whereas genes implicated in signal transduction are primarily of eukaryotic origin, and many of the genes for defense and repair functions, in particular nucleases, seem to be of bacterial or bacteriophages origin. Altogether, the findings report- 
ed by Boyer et al. led them to hypothesize that numerous Marseillevirus ORFs were acquired from many different sources through LGT and that the mosaicism exhibited by the Marseillevirus genome could have resulted from gene exchange with sympatric amoeba-infecting bacteria and viruses that have an intra-amoebal lifestyle, as well as with their amoebal host [11]. LGT may be common, as suggested by several cases where related genes seemed to be acquired from independent sources by Marseillevirus and Mimivirus [11].

The substantial flow of genes due to LGT that has been detected in amoeba-associated viruses prompts the desire to understand the mechanisms of bacterial-like gene acquisition by APMV. It is assumed that free-living amoebae harbor a spectrum of pathogenic and non-pathogenic bacteria [69]. Although the current knowledge of the replication cycle of APMV and other NCLDVs is very limited [70], it is conceivable that amoebae may promote gene exchange between bacterial and viral DNA during their replication in a same cell compartment $[8,69,71]$. One possible mechanism for LGT between bacterial and APMV genomes may be suggested by the replication mode of poxviruses, other NCLDVs [72]. Indeed, replicating poxviruses have been found to display very high levels of homologous recombination that requires little sequence homology [73] and appears to occur predominantly at the ends of the viral genome [74, 75]. Interestingly, similar recombination frequencies have been noted in phycodnaviruses [76]. Another mechanism may be similar to that observed during recombination in Herpesviridae, which are also large DNA viruses [77]. This process involves a DNA-binding protein and an exonuclease, and replication is unnecessary. Alternatively, it has been proposed that recombination may be promoted by an enzyme similar to the topoisomerase IB of the vaccinia virus $[51,78]$. In support of this, a DNA topoisomerase IB encoded by the APMV genome has been produced, purified and characterized [79]. Interestingly, an evolutionary and functional relationship was demonstrated among the APMV, poxvirus, and bacterial topoisomerase IB proteins, and dissemination of an ancestral bacterial/viral enzyme by LGT was hypothesized.

The survival and life cycle of giant viruses in amoebae may be the cornerstone of their gene repertoire (fig. 3). Free-living amoebae of the genus Acanthamoeba are ubiquitous in the environment and can be isolated from air, soil and water of various sources [80, 81]. Amoebae are wild phagocytes that ingest any particle larger than $0.5 \mu \mathrm{m}$ [82]. They harbor a variety of intracellular bacteria (either transiently or for long periods), including Chla- mydia, Bacteroidetes, Actinobacteria, Firmicutes and Proteobacteria [69]. Several mimiviral ORFs have homologs in bacterial species that are common inhabitants of amoebae [31]. For instance, two ORFs corresponding to a Zndependant alcohol dehydrogenase and an outer-membrane lipoprotein seem to have homologs in Legionella pneumophila and Campylobacter spp., respectively. In the genome of Marseillevirus, the most recently described amoeba-infecting giant virus, $41 \%$ of ORFs exhibit the highest sequence similarities to homologs found in viruses, bacteria, archaea and eukaryotes, and it has been suggested that the large proportion of chimeric genes observed in Marseillevirus might have resulted from LGT with sympatric intra-amoebal bacteria and viruses [11]. A recent work has underscored the observation that amoebal endosymbionts appear to have larger genomes than their relatives [83], in contrast to other intracellular bacteria whose lifestyle is associated with genome reduction $[84,85]$. Thus, Moliner et al. reported that the Legionella drancourtii genome is larger than those sequenced from L. pneumophila strains (4.2 Mb vs. $3.5 \mathrm{Mb}$ on average) $[83,86,87]$. Along the same lines, the genome of Rickettsia bellii is the largest among Rickettsia species [71], and the genomes of Candidatus 'Protochlamydia amoebophila' and of Parachlamydia acanthamoebae are approximately twice as large as those of pathogenic Chlamydia species [88, 89]. Among viruses, APMV and Marseillevirus have the largest and fifth-largest genomes, respectively $[9,11]$. Therefore, in all those previous examples, the intra-amoebal lifestyle has been positively associated with genome size. This may be explained by possible foraging of genes by sympatric microorganisms due to the promiscuity between their replicating genomes. In contrast, the lifestyle of obligate intracellular bacteria in other eukaryotic cells limits such genetic promiscuity and the concomitant capacity to acquire foreign genes (fig. 3). The work by Moliner et al. further provides a very good illustration of how the intra-amoebal lifestyle might enable the exchange of genes across the boundaries of phyla, kingdoms, and even domains, and that some of these genes might have ancient origins [83]. Thus, a sterol delta-7 reductase was detected among orthologous proteins of Candidatus 'Protochlamydia amoebophila', $L$. drancourtii, and Coxiella burnetii, three amoeba-resistant bacteria, but not in other related species. Furthermore, this protein is closely related to sterol delta-7 reductases of Viridiplantae, which led to the hypothesis that its gene may have been acquired by amoeba-resistant bacteria following a transfer from Viridiplantae. Other studies have pointed out the putative role of amoebae in gene ex- 
changes between sympatric intra-cellular pathogens. For instance, ATP/ADP translocases were found to be a common feature of obligate intra-cellular amoebal symbionts related to Chlamydiae and Rickettsiae [90]. Additionally, it has been suggested that amoeba-like ancestral protozoa might have served as a genetic 'melting pot' where the ancestors of Rickettsia and other bacteria exchanged genes that may have permitted their adaptation to an intracellular lifestyle in eukaryotic cells [71]. In contrast, species living in restricted environments and lacking mechanisms of gene exchange may have evolved with considerably less variation. An example is the obligate intracellular endosymbiont of aphids, Buchnera aphidicola, in which no chromosome rearrangements or gene acquisitions have occurred in the past 50-70 million years [91]. Furthermore, a massive comparative analysis of 317 genomes of bacteria with different lifestyles recently demonstrated a convergent evolution for obligate intracellular bacteria, which included massive gene loss as a driving force in the adaptation of parasites to eukaryote cells [92].

The remarkably extensive and diverse gene content of amoeba-associated giant viruses may provide them several benefits. L. pneumophila has acquired by LGT eukaryotic genes involved in a variety of cell functions, including two serine/threonine protein kinases [93]. Such enzymes have been showed to inhibit phagosome-lysosome fusion in several pathogens, promoting their intracellular survival and the disruption of host defenses by interfering with eukaryotic signal transduction [94]. In this view, the presence in the Mimivirus genome of three serine/threonine protein kinases acquired from its amoebal hosts and a RAS GTPase suggest that APMV may regulate the host cell cycle for its own benefit [31]. Furthermore, in addition to the fact that Acanthamoeba species can, themselves, infect a large spectrum of mammals, including humans [95], their role as 'Trojan horses' has been discussed $[81,96]$. Thus, most bacteria that multiply in amoebae are human pathogens [97]. The large gene content of giant viruses that multiply in amoebae may allow them to survive and be pathogenic outside their host after this latter has played its role of environmental reservoir and gene-transfer vehicle [69]. Ghigo et al. demonstrated that APMV could enter macrophages by internalization, a process that closely resembles that mediated by amoeba [50, 98]. This suggests that amoebae may promote the adaptation of some microorganisms to macrophages. For instance, L. pneumophila, C. burnetii and Parachlamydiaceae are resistant to amoebae and have been shown to be pathogens for macrophages [98, 99]. The finding that the intracellular survival strategies of
Cryptococcus neoformans in amoebae and macrophages are very similar also suggests that selective pressures placed by amoebae on microorganisms may be a key factor in the maintenance of their virulence characteristics for animal hosts [100]. These data further suggest a pathogenic role for APMV and are in agreement with findings that support a causative role for it in pneumonia [101105]. The detection in Mimivirus and Marseillevirus of LGTs involving different eukaryotic sources and the relative paucity of genes of Acanthamoeba origin further suggest that these viruses may have a larger spectrum of hosts than was historically thought and that they may have changed hosts in the course of their evolution $[9,11$, 48]. In agreement with this hypothesis, very recent findings suggest that Mimivirus relatives may be very ubiquitous in the biosphere, and microalgae and modern sponges might be hosts to yet unidentified members of the Mimiviridae [106, 107]. Finally, previous findings have underscored that amoebae are unique host cells where bacteria and viruses are in proximity. Besides exchanging genes, these microorganisms might compete to survive and multiply. Amoebae might therefore represent a battlefield where sympatric microorganisms struggle for life with their gene armories. In this setting, possessing a plethoric and/or chimeric gene repertoire might be very beneficial to intra-amoebal giant viruses because, as proposed in the Red Queen hypothesis, no species can ever win in such an environment as the amoebal arena [108].

\section{Duplicated Genes}

Besides LGT, it has been suggested that gene and genome duplication played a major role in shaping the APMV genome [109]. Such events were defined several decades ago and are associated with the emergence of new gene material with different fates [110]. Duplicated genes can evolve towards nonfunctionality, with degeneration into a pseudogene and possible elimination, or towards sub- or neo-functionalization. The divergence of duplicated genes may be very important in evolution, so much so that the homology between many genes with common ancestry can no longer be detected [111]. Suhre determined that about one-third of APMV genes have one or more paralogs in the genome [109]. Depending on the choice of the e-value cutoff, between 26.3 and $35.0 \%$ of APMV genes were found to have at least one paralog. The number of paralogs was estimated to be as high as 66 , and the orientation and location of gene duplication events appear to be non-random. Indeed, duplicated genes are inserted about twice as frequently in the parallel orientation as in the antiparallel orientation, with re- 
spect to the coding direction of the matching gene (20.2 vs. $11.7 \%$ ), and the proportion of duplications that are localized to the same half of the genome is $79 \%$ for duplications in cis versus 39\% for duplications in trans. Interestingly, among the major families of paralogs, several showed homology, although often remote or only putative, with genes encoding proteins that are likely to play a role in virus-host interactions by interfering with important host processes such as transcription, protein degradation, or cellular regulatory processes. One such family includes proteins with a Pfam F-box domain, which is a receptor that serves as a link between a target protein and a ubiquitin-conjugating enzyme. The largest paralogous family corresponds to ankyrin repeat-containing proteins that might have a structural role. Finally, aside from gene duplication, Suhre described data that suggest the occurrence of segmental duplication implicating a large part of the $5^{\prime}$-end and $3^{\prime}$-end of the genome. The large number of gene duplications in the APMV genome demonstrated in the study of Suhre lends support to the theory that duplication events contributed substantially to its large size, without a substantial requirement of LGT for the acquisition of genes. As a matter of fact, the gene duplication rate of APMV is within the range of frequencies observed in the three domains of life. The hypothesis that duplicated genes interfere with host processes may be compatible with the accretion and fixation of the large gene content of the APMV genome because the latter would allow APMV to mimic large microbial prey for amoebae. Supporting this hypothesis, a log-linear correlation could be identified for APMV and other large DNA viruses between the number of paralogous genes and the number of predicted genes within the genome [109].

\section{ORFans in Mimivirus, Marseillevirus and the Sputnik} Virophage

ORFans refer to genes without detectable homologs in sequence databases [112]. A challenging feature of the genomes of APMV, Marseillevirus and the Sputnik virophage are that a substantial part of their predicted coding genes do not exhibit clear homology to proteins of known function $[8,9,11]$. Furthermore, $39 \%$ of APMV ORFs and 13 of Sputnik's 21 predicted genes do not clearly match any sequence found in databases $[8,9]$, whereas sequence similarity and conserved domain searches against NCBI databases identified significant matches and/or conserved domains for only 188 of the 457 Marseillevirus predicted ORFs. The substantial proportion of ORFans in the genomes of amoeba-associated viruses underscores the fact that our sequence databases, although rap- idly growing, may still cover only a small fraction of the pangenome of our biosphere. As ORFans of amoeba-associated giant viruses are the topic of another review in this journal issue, they are not described here.

\section{Conclusion}

In a recent correspondence letter on the complexity of the virus world, Koonin et al. stated that 'the virus world is a dynamic network of relationships in which genes have diverse, variously intertwined histories' [113]. This paradigm seems to apply perfectly to the giant viruses isolated from amoebae. The genomes of Mimivirus and Marseillevirus contain core genes of NCLDVs but also genes of various origins in the three domains of life. Thus, they are made up of a backbone of genes upon which is grafted a complex, chimeric, dispensable genome acquired largely through LGT or gene duplication events. Moreover, the genomes of Mimivirus, Marseillevirus and Sputnik contain many ORFans. Gene accretion, leading to unexpectedly large repertoires of genes of various and sometimes remote origins, probably occurs as a consequence of an intra-amoebal lifestyle, which enables the sharing of genes between sympatric bacteria, viruses and eukaryotes. This provides amoeba-associated giant viruses with gene armories that may help them out-compete other microorganisms present in their hosts. Finally, the remarkably extensive and diverse gene content of Mimivirus and Marseillevirus may enable them to venture outside the amoebae. In support of this, Mimivirus has been shown to be internalized by macrophages, leading to productive replication [98], and several studies implicate this virus as a possible causative agent of pneumonia [101-105]. Altogether, the findings reviewed herein are a powerful incentive to find and study other amoeba-associated giant viruses, so that we may gain a better understanding of their pangenome. 


\section{References}

1 Fuhrman JA: Marine viruses and their biogeochemical and ecological effects. Nature 1999;399:541-548.

$\checkmark 2$ Suttle CA: Viruses in the sea. Nature 2005; 437:356-361.

73 Woese C: The universal ancestor. Proc Natl Acad Sci USA 1998;95:6854-6859.

-4 Woese CR, Kandler O, Wheelis ML: Towards a natural system of organisms: proposal for the domains Archaea, Bacteria, and Eucarya. Proc Natl Acad Sci USA 1990;87:4576-4579.

5 van Regenmortel MHV: 7th report of the International Committee on taxonomy of Viruses. San Diego, Academic Press, 2000, pp 3-16.

6 Lwoff A: The concept of virus. J Gen Microbiol 1957;17:239-253.

7 Raoult D, La Scola B, Birtles R: The discovery and characterization of Mimivirus, the largest known virus and putative pneumonia agent. Clin Infect Dis 2007;45:95-102.

$>8$ La Scola B, Desnues C, Pagnier I, Robert C, Barrassi L, Fournous G, Merchat M, SuzanMonti M, Forterre P, Koonin E, Raoult D: The virophage as a unique parasite of the giant mimivirus. Nature 2008;455:100-104.

$>9$ Raoult D, Audic S, Robert C, Abergel C, Renesto P, Ogata H, La Scola B, Suzan M, Claverie JM: The 1.2-megabase genome sequence of Mimivirus. Science 2004;306: 1344-1350.

$>10$ La Scola B, Audic S, Robert C, Jungang L, de Lamballerie X, Drancourt M, Birtles R, Claverie JM, Raoult $\mathrm{D}$ : A giant virus in amoebae. Science 2003;299:2033.

$\checkmark 11$ Boyer L, Yutin N, Pagnier I, Barassi L, Fournous G, Espinosa L, Robert C, Azza S, Sun S, Rossmann MG, Suzan-Monti M, La Scola B, Koonin EV, Raoult D: Giant Marseillevirus highlights the role of amoebae as a melting pot in emergence of chimeric microorganisms. Proc Natl Acad Sci USA 2009;106: 21848-21853.

$\checkmark 12$ Iyer LM, Balaji S, Koonin EV, Aravind L: Evolutionary genomics of nucleo-cytoplasmic large DNA viruses. Virus Res 2006;117: 156-184.

13 Koonin EV: Virology: Gulliver among the Lilliputians. Curr Biol 2005;15:R167-R169.

$\checkmark 14$ Claverie JM, Abergel C: Mimivirus and its virophage. Annu Rev Genet 2009;43:49-66.

15 Yamada T, Onimatsu H, Van Etten JL: Chlorella viruses. Adv Virus Res 2006;66:293336.

-16 Delaroque N, Boland W, Muller DG, Knippers R: Comparisons of two large phaeoviral genomes and evolutionary implications. J Mol Evol 2003;57:613-622.

-17 Freeman MJ, Plasterer NT, Smith FT, Mohr CS: Patterns of genome organization in bacteria. Science 1998;279:1827.

18 Galperin MY, Koonin EV: 'Conserved hypothetical' proteins: prioritization of targets for experimental study. Nucleic Acids Res 2004; 32:5452-5463.
19 Koonin EV: Orthologs, paralogs, and evolutionary genomics. Annu Rev Genet 2005;39: 309-338.

20 Fitzgerald LA, Graves MV, Li X, Feldblyum T, Nierman WC, Van Etten JL: Sequence and annotation of the $369-\mathrm{kb}$ NY-2A and the $345-\mathrm{kb}$ AR158 viruses that infect Chlorella NC64A. Virology 2007;358:472-484.

21 Byrne D, Grzela R, Lartigue A, Audic S, Chenivesse S, Encinas S, Claverie JM, Abergel C: The polyadenylation site of Mimivirus transcripts obeys a stringent 'hairpin rule'. Genome Res 2009;19:1233-1242.

22 Suhre K, Audic S, Claverie JM: Mimivirus gene promoters exhibit an unprecedented conservation among all eukaryotes. Proc Natl Acad Sci USA 2005;102:14689-14693.

23 Wilson WH, Schroeder DC, Allen MJ, Holden MT, Parkhill J, Barrell BG, Churcher C, Hamlin N, Mungall K, Norbertczak H, Quail MA, Price C, Rabbinowitsch E, Walker D, Craigon M, Roy D, Ghazal P: Complete genome sequence and lytic phase transcription profile of a Coccolithovirus. Science 2005; 309:1090-1092.

24 Hutson MS, Duke T, Viovy JL: Two-dimensional motion of DNA bands during $120^{\circ}$ pulsed-field gel electrophoresis. I. Effect of molecular weight. Biopolymers 1995;35: 297-306.

$\checkmark 25$ Claverie JM, Ogata H, Audic S, Abergel C, Suhre K, Fournier PE: Mimivirus and the emerging concept of 'giant' virus. Virus Res 2006;117:133-144.

-26 Koonin EV, Senkevich TG, Dolja VV: The ancient Virus World and evolution of cells Biol Direct 2006; 1:29.

27 Iyer LM, Aravind L, Koonin EV: Common origin of four diverse families of large eukaryotic DNA viruses. J Virol 2001;75: 11720-11734

28 Yutin N, Wolf YI, Raoult D, Koonin EV: Eukaryotic large nucleo-cytoplasmic DNA viruses: clusters of orthologous genes and reconstruction of viral genome evolution. Virol J 2009;6:223.

29 Awadalla P: The evolutionary genomics of pathogen recombination. Nat Rev Genet 2003;4:50-60.

30 Moreira D, Lopez-Garcia P: Ten reasons to exclude viruses from the tree of life. Nat Rev Microbiol 2009;7:306-311.

31 Moreira D, Brochier-Armanet C: Giant viruses, giant chimeras: the multiple evolutionary histories of Mimivirus genes. BMC Evol Biol 2008;8:12.

32 Moreira D, Lopez-Garcia P: Comment on 'The 1.2-megabase genome sequence of Mimivirus'. Science 2005;308:1114.

33 Andersson JO: Lateral gene transfer in eukaryotes. Cell Mol Life Sci 2005;62:11821197.

34 Lawrence JG, Ochman H: Amelioration of bacterial genomes: rates of change and exchange. J Mol Evol 1997;44:383-397.
35 Jain R, Rivera MC, Moore JE, Lake JA: Horizontal gene transfer accelerates genome innovation and evolution. Mol Biol Evol 2003; 20:1598-1602.

36 Canchaya C, Fournous G, Chibani-Chennoufi S, Dillmann ML, Brussow $\mathrm{H}$ : Phage as agents of lateral gene transfer. Curr Opin Microbiol 2003;6:417-424.

37 Filee J, Bapteste E, Susko E, Krisch HM: A selective barrier to horizontal gene transfer in the T4-type bacteriophages that has preserved a core genome with the viral replication and structural genes. Mol Biol Evol 2006;23:1688-1696.

38 Filee J, Tetart F, Suttle CA, Krisch HM: Marine T4-type bacteriophages, a ubiquitous component of the dark matter of the biosphere. Proc Natl Acad Sci USA 2005;102: 12471-12476

39 Lindell D, Sullivan MB, Johnson ZI, Tolonen AC, Rohwer F, Chisholm SW: Transfer of photosynthesis genes to and from Prochlorococcus viruses. Proc Natl Acad Sci USA 2004;101:11013-11018

-40 Millard A, Clokie MR, Shub DA, Mann NH Genetic organization of the psbAD region in phages infecting marine Synechococcus strains. Proc Natl Acad Sci USA 2004;101: 11007-11012

41 McGeoch DJ, Rixon FJ, Davison AJ: Topics in herpesvirus genomics and evolution. Virus Res 2006;117:90-104.

42 Alcami A, Koszinowski UH: Viral mechanisms of immune evasion. Trends Microbiol 2000;8:410-418.

-43 McFadden G, Murphy PM: Host-related immunomodulators encoded by poxviruses and herpesviruses. Curr Opin Microbiol 2000;3:371-378.

44 Moore PS, Boshoff C, Weiss RA, Chang Y: Molecular mimicry of human cytokine and cytokine response pathway genes by KSHV. Science 1996;274:1739-1744.

45 Fu M, Deng R, Wang J, Wang X: Detection and analysis of horizontal gene transfer in herpesvirus. Virus Res 2008;131:65-76.

46 Filee J, Pouget N, Chandler M: Phylogenetic evidence for extensive lateral acquisition of cellular genes by nucleocytoplasmic large DNA viruses. BMC Evol Biol 2008;8:320

-47 Esposito JJ, Sammons SA, Frace AM, Osborne JD, Olsen-Rasmussen M, Zhang M, Govil D, Damon IK, Kline R, Laker M, Li Y, Smith GL, Meyer H, Leduc JW, Wohlhueter RM: Genome sequence diversity and clues to the evolution of variola (smallpox) virus. Science 2006;313:807-812.

48 Filee J: Lateral gene transfer, lineage-specific gene expansion and the evolution of Nucleo Cytoplasmic Large DNA viruses. J Invertebr Pathol 2009;101:169-171.

49 Ogata H, Abergel C, Raoult D, Claverie JM Response to Comment on 'The 1.2-Megabase genome sequence of Mimivirus'. Science 2005;308:1114b. 
50 Suzan-Monti M, La Scola B, Raoult D: Genomic and evolutionary aspects of Mimivirus. Virus Res 2006;117:145-155.

51 Filee J, Siguier P, Chandler M: I am what I eat and I eat what I am: acquisition of bacterial genes by giant viruses. Trends Genet 2007; 23:10-15.

-52 Tatusov RL, Fedorova ND, Jackson JD, Jacobs AR, Kiryutin B, Koonin EV, Krylov DM, Mazumder R, Mekhedov SL, Nikolskaya AN, Rao BS, Smirnov S, Sverdlov AV, Vasudevan S, Wolf YI, Yin JJ, Natale DA: The COG database: an updated version includes eukaryotes. BMC Bioinformatics 2003;4:41.

53 Dyková I, Fiala I, Lom J, Lukeš J: Perkinsiella amoebae-like endosymbionts of Neoparamoeba spp., relatives of the kinetoplastid Ichthyobodo. Eur J Protistol 2003;39:37-52.

- 54 Frost LS, Leplae R, Summers AO, Toussaint A: Mobile genetic elements: the agents of open source evolution. Nat Rev Microbiol 2005;3:722-732.

-55 Ton-Hoang B, Guynet C, Ronning DR, Cointin-Marty B, Dyda F, Chandler M: Transposition of ISHp608, member of an unusual family of bacterial insertion sequences. EMBO J 2005;24:3325-3338.

56 Guhan N, Muniyappa K: Structural and functional characteristics of homing endonucleases. Crit Rev Biochem Mol Biol 2003 38:199-248.

57 Ogata H, Raoult D, Claverie JM: A new example of viral intein in Mimivirus. Virol J $2005 ; 2: 8$.

- 58 Hirata R, Ohsumk Y, Nakano A, Kawasaki H, Suzuki K, Anraku Y: Molecular structure of a gene, VMA1, encoding the catalytic subunit of $\mathrm{H}(+)$-translocating adenosine triphosphatase from vacuolar membranes of Saccharomyces cerevisiae. J Biol Chem 1990; 265:6726-6733.

59 Kane PM, Yamashiro CT, Wolczyk DF, Neff N, Goebl M, Stevens TH: Protein splicing converts the yeast TFP1 gene product to the $69-\mathrm{kD}$ subunit of the vacuolar $\mathrm{H}(+)$-adenosine triphosphatase. Science 1990;250:651657.

-60 Pietrokovski S: Identification of a virus intein and a possible variation in the proteinsplicing reaction. Curr Biol 1998;8:R634R635.

-61 Weynberg KD, Allen MJ, Ashelford K, Scanlan DJ, Wilson WH: From small hosts come big viruses: the complete genome of a second Ostreococcus tauri virus, OtV-1. Environ Microbiol 2009;11:2821-2839.

62 Williamson SJ, Rusch DB, Yooseph S, Halpern AL, Heidelberg KB, Glass JI, AndrewsPfannkoch C, Fadrosh D, Miller CS, Sutton G, Frazier M, Venter JC: The Sorcerer II Global Ocean Sampling Expedition: metagenomic characterization of viruses within aquatic microbial samples. PLoS One 2008; 3:e1456.
63 Iyer LM, Makarova KS, Koonin EV, Aravind L: Comparative genomics of the FtsK-HerA superfamily of pumping ATPases: implications for the origins of chromosome segregation, cell division and viral capsid packaging. Nucleic Acids Res 2004;32:5260-5279.

64 De Silva FS, Moss B: Origin-independent plasmid replication occurs in vaccinia virus cytoplasmic factories and requires all five known poxvirus replication factors. Virol J 2005;2:23.

65 Rojo G, Garcia-Beato R, Vinuela E, Salas ML, Salas J: Replication of African swine fever virus DNA in infected cells. Virology 1999; 257:524-536

66 Gubbels MJ, Vaishnava S, Boot N, Dubremetz JF, Striepen B: A MORN-repeat protein is a dynamic component of the Toxoplasma gondii cell division apparatus. J Cell Sci 2006;119:2236-2245.

67 Cheng CH, Liu SM, Chow TY, Hsiao YY, Wang DP, Huang JJ, Chen HH: Analysis of the complete genome sequence of the $\mathrm{Hz}-1$ virus suggests that it is related to members of the Baculoviridae. J Virol 2002;76:90249034.

68 Ibrahim AMA, Choi JY, Je YH, Kim Y: Structure and expression profile of two putative Cotesia plutellae bracovirus genes (CpBV-H4 and CpBV-E94\{alpha\}) in parasitized Plutel la xylostella. J Asia Pacific Entomol 2005;8: 359-366.

69 Horn M, Wagner M: Bacterial endosymbionts of free-living amoebae. J Eukaryot Microbiol 2004;51:509-514.

70 Suzan-Monti M, La Scola B, Barrassi L, Espinosa L, Raoult D: Ultrastructural characterization of the giant volcano-like virus factory of Acanthamoeba polyphaga Mimivirus. PLoS One 2007;2:e328.

-71 Ogata H, La Scola B, Audic S, Renesto P, Blanc G, Robert C, Fournier PE, Claverie JM, Raoult D: Genome sequence of Rickettsia bellii illuminates the role of amoebae in gene exchanges between intracellular pathogens. PLoS Genet 2006;2:e76.

72 Yao XD, Evans DH: Effects of DNA structure and homology length on vaccinia virus recombination. J Virol 2001;75:6923-6932.

73 Willer DO, Yao XD, Mann MJ, Evans DH: In vitro concatemer formation catalyzed by vaccinia virus DNA polymerase. Virology 2000;278:562-569.

74 Ball LA: High-frequency homologous recombination in vaccinia virus DNA. J Virol 1987;61:1788-1795

75 Evans DH, Stuart D, McFadden G: High levels of genetic recombination among cotransfected plasmid DNAs in poxvirus-infected mammalian cells. J Virol 1988;62:367-375.

76 Tessman I: Genetic recombination of the DNA plant virus PBCV1 in a Chlorella-like alga. Virology 1985;145:319-322.
7 Reuven NB, Willcox S, Griffith JD, Weller SK: Catalysis of strand exchange by the HSV$1 \mathrm{UL} 12$ and ICP8 proteins: potent ICP8 recombinase activity is revealed upon resection of dsDNA substrate by nuclease. J Mol Biol 2004;342:57-71.

78 Cheng C, Shuman S: Recombinogenic flap ligation pathway for intrinsic repair of topoisomerase IB-induced double-strand breaks. Mol Cell Biol 2000;20:8059-8068.

79 Benarroch D, Claverie JM, Raoult D, Shuman S: Characterization of mimivirus DNA topoisomerase IB suggests horizontal gene transfer between eukaryal viruses and bacteria. J Virol 2006;80:314-321.

80 Rodriguez-Zaragoza S: Ecology of free-living amoebae. Crit Rev Microbiol 1994;20: 225-241.

-81 Mattana A, Serra C, Mariotti E, Delogu G, Fiori PL, Cappuccinelli P: Acanthamoeba castellanii promotion of in vitro survival and transmission of coxsackie b3 viruses. Eukaryot Cell 2006;5:665-671.

-82 Audic S, Robert C, Campagna B, Parinello H Claverie JM, Raoult D, Drancourt M: Genome analysis of Minibacterium massiliensis highlights the convergent evolution of water-living bacteria. PLoS Genet 2007; 3:e138.

83 Moliner C, Raoult D, Fournier PE: Evidence that the intra-amoebal Legionella drancourtii acquired a sterol reductase gene from eukaryotes. BMC Res Notes 2009;2:51.

84 Boussau B, Karlberg EO, Frank AC, Legault BA, Andersson SG: Computational inference of scenarios for alpha-proteobacterial genome evolution. Proc Natl Acad Sci USA 2004;101:9722-9727.

85 Sakharkar KR, Dhar PK, Chow VT: Genome reduction in prokaryotic obligatory intracellular parasites of humans: a comparative analysis. Int J Syst Evol Microbiol 2004;54: 1937-1941.

86 Chien M, Morozova I, Shi S, Sheng H, Chen J, Gomez SM, Asamani G, Hill K, Nuara J, Feder M, Rineer J, Greenberg JJ, Steshenko V, Park SH, Zhao B, Teplitskaya E, Edwards JR, Pampou S, Georghiou A, Chou IC, Iannuccilli W, Ulz ME, Kim DH, Geringer-Sameth A, Goldsberry C, Morozov P, Fischer SG, Segal G, Qu X, Rzhetsky A, Zhang P, Cayanis E, De Jong PJ, Ju J, Kalachikov S, Shuman HA, Russo JJ: The genomic sequence of the accidental pathogen Legionella pneumophila. Science 2004;305:1966-1968.

- 87 Cazalet C, Rusniok C, Bruggemann H, Zidane N, Magnier A, Ma L, Tichit M, Jarraud $S$, Bouchier $C$, Vandenesch F, Kunst F, Etienne J, Glaser P, Buchrieser C: Evidence in the Legionella pneumophila genome for exploitation of host cell functions and high genome plasticity. Nat Genet 2004;36:11651173. 
88 Horn M, Collingro A, Schmitz-Esser S, Beier CL, Purkhold U, Fartmann B, Brandt P, Nyakatura GJ, Droege M, Frishman D, Rattei T, Mewes HW, Wagner M: Illuminating the evolutionary history of chlamydiae. Science 2004;304:728-730.

89 Greub G, Kebbi-Beghdadi C, Bertelli C, Collyn F, Riederer BM, Yersin C, Croxatto A, Raoult D: High throughput sequencing and proteomics to identify immunogenic proteins of a new pathogen: the dirty genome approach. PLoS One 2009; 4:e8423.

90 Schmitz-Esser S, Linka N, Collingro A, Beier CL, Neuhaus HE, Wagner M, Horn M: ATP/ ADP translocases: a common feature of obligate intracellular amoebal symbionts related to Chlamydiae and Rickettsiae. J Bacteriol 2004;186:683-691.

-91 Tamas I, Klasson L, Canback B, Naslund AK, Eriksson AS, Wernegreen JJ, Sandstrom JP, Moran NA, Andersson SG: 50 million years of genomic stasis in endosymbiotic bacteria. Science 2002;296:2376-2379.

-92 Merhej V, Royer-Carenzi M, Pontarotti P, Raoult D: Massive comparative genomic analysis reveals convergent evolution of specialized bacteria. Biol Direct 2009;4:13.

-93 Bruggemann H, Cazalet C, Buchrieser C: Adaptation of Legionella pneumophila to the host environment: role of protein secretion, effectors and eukaryotic-like proteins. Curr Opin Microbiol 2006;9:86-94.

\$4 Walburger A, Koul A, Ferrari G, Nguyen L, Prescianotto-Baschong C, Huygen K, Klebl B, Thompson C, Bacher G, Pieters J: Protein kinase $\mathrm{G}$ from pathogenic mycobacteria promotes survival within macrophages. Science 2004;304:1800-1804.
95 Meersseman W, Lagrou K, Sciot R, de JJ, Haberler C, Walochnik J, Peetermans WE, van WE: Rapidly fatal Acanthamoeba encephalitis and treatment of cryoglobulinemia. Emerg Infect Dis 2007;13:469-471.

$\$ 96$ Abu KY, Gao LY, Stone BJ, Venkataraman C, Harb OS: Invasion of protozoa by Legionella pneumophila and its role in bacterial ecology and pathogenesis. Appl Environ Microbiol 1998;64:3127-3133.

97 Barker J, Brown MR: Trojan horses of the microbial world: protozoa and the survival of bacterial pathogens in the environment. Microbiology 1994;140:1253-1259.

98 Ghigo E, Kartenbeck J, Lien P, Pelkmans L, Capo C, Mege JL, Raoult D: Ameobal pathogen Mimivirus infects macrophages through phagocytosis. PLoS Pathog 2008; 4:e1000087.

99 Greub G, Raoult D: Microorganisms resistant to free-living amoebae. Clin Microbiol Rev 2004; 17:413-433.

100 Steenbergen JN, Shuman HA, Casadevall A: Cryptococcus neoformans interactions with amoebae suggest an explanation for its virulence and intracellular pathogenic strategy in macrophages. Proc Natl Acad Sci USA 2001;98:15245-15250.

101 La Scola B, Marrie TJ, Auffray JP, Raoult D: Mimivirus in pneumonia patients. Emerg Infect Dis 2005;11:449-452.

102 Berger P, Papazian L, Drancourt M, La Scola B, Auffray JP, Raoult D: Ameba-associated microorganisms and diagnosis of nosocomial pneumonia. Emerg Infect Dis 2006;12:248-255.
03 Raoult D, Renesto P, Brouqui P: Laboratory infection of a technician by mimivirus. Ann Intern Med 2006;144:702-703.

104 Khan M, La Scola B, Lepidi H, Raoult D: Pneumonia in mice inoculated experimentally with Acanthamoeba polyphaga mimivirus. Microb Pathog 2007;42:56-61.

105 Vincent A, La Scola B, Forel JM, Pauly V, Raoult D, Papazian L: Clinical significance of a positive serology for mimivirus in patients presenting a suspicion of ventilatorassociated pneumonia. Crit Care Med 2009;37:111-118.

106 Monier A, Larsen JB, Sandaa RA, Bratbak G, Claverie JM, Ogata H: Marine mimivirus relatives are probably large algal viruses. Virol J 2008;5:12.

107 Claverie JM, Grzela R, Lartigue A, Bernadac A, Nitsche S, Vacelet J, Ogata H, Abergel C: Mimivirus and Mimiviridae: giant viruses with an increasing number of potential hosts, including corals and sponges. J Invertebr Pathol 2009;101:172-180.

108 Van Valen L: A new evolutionary law. Evolutionary Theory 1973;1:1-30.

109 Suhre K: Gene and genome duplication in Acanthamoeba polyphaga Mimivirus. J Virol 2005;79:14095-14101.

10 Zhang J: Evolution by gene duplication: an update. Trends Ecol Evol 2003;18:292-298.

111 Hurles M: Gene duplication: the genomic trade in spare parts. PLoS Biol 2004;2:E206.

112 Fischer D, Eisenberg D: Finding families for genomic ORFans. Bioinformatics 1999;15: 759-762.

113 Koonin EV, Wolf YI, Nagasaki K, Dolja VV: The complexity of the virus world. Nat Rev Microbiol 2009, E-pub ahead of print. 